\title{
Minimization of Power Losses in Transmission Lines
}

\author{
${ }^{1}$ Anyaka Boniface Onyemaechi, ${ }^{2}$ Olawoore Olaoluwa Isaac \\ 1, 2, Department Of Electrical Engineering University Of Nigeria,Nsukka, Nigeria
}

\begin{abstract}
Availability Of Electric Power Has Been The Most Powerful Vehicle For Facilitating Economic, Industrial And Social Developments Of Any Nation. Electric Power In Nigeria Is Transmitted By Means Of Transmission Lines Which Deliver Bulk Power From Generating Stations To Load Centres And Consumers. For Electric Power To Get To The Final Consumers In Proper Form And Good Quality, Losses Along The Lines Must Be Reduced To The Barest Minimum. In This Research Work Capacitor Was Used To Generate Capacitive Power To Cancel The Inductive Reactance Of The System As Such Compensating For Power Losses Along Transmission Line Such That Power Generated From The Source Could Get To The End Users With Minimized Or No Loss.
\end{abstract}

Key Words: Minimization, Power Losses, Transmission Line, Reactive Load, Capacitor.

\section{Introduction}

Energy Is A Basic Necessity For The Economic Development Of A Nation. There Are Different Forms Of Energy, But The Most Important Form Is The Electrical Energy. A Modern And Civilized Society Is So Much Dependent On The Use Of Electrical Energy. Activities Relating To The Generation, Transmission And Distribution Of Electrical Energy Have To Be Given The Highest Priority In The National Planning Process Of Any Nation Because Of The Importance Of Electrical Energy To The Economic And Social Development Of The Society. In Fact, The Greater The Per Capital Consumption Of Electrical Energy In A Country, The Higher The Standard Of Living Of Its People. Therefore, The Advancement Of A Country Is Measured In Terms Of Its Per Capital Consumption Of Electrical Energy. Power Plants' Planning In A Way To Meet The Power Network Load Demand Is One Of The Most Important And Essential Issues In Power Systems. Since Transmission Lines Connect Generating Plants And Substations In Power Network, The Analysis, Computation And Reduction Of Transmission Losses In These Power Networks Are Of Great Concern To Engineers. A Lot Of Research Works Have Been Carried Out On The Above Listed Aspects. There Was A Comparison Between The Corona Power Loss Associated With HVDC Transmission Lines And The Ohmic Power Loss [1]. The Corona Power Loss And Ohmic Power Loss Were Measured And Computed For Different Transmission Line Configurations And Under Fair Weather And Rainy Conditions. It Was Pointed Out In The Work That The General Trend Of Neglecting The Corona Power Loss Is Not Always Valid. It Was Found From The Comparison That, When The Transmission Line Is Moderately Or Lightly Loaded, The Percentage Of Corona Power Loss To Ohmic Power Loss Could Reach Up To One Hundred Percent Especially If The Transmission Line Is Operating At A Voltage Well Above The Corona Onset Value. This Percentage Is Also Found To Increase Substantially Under Rainy Conditions. Finally, It Was Also Discovered That, The Ratio Of Corona To Ohmic Power Loss Decreases With Increasing Number Of Bundles. There Has Been A Research On Loss Minimization Using Optimal Power Flow Based On Swarm Intelligences [6]. Consideration Of Multi-Objective Optimization Methods For Power Loss Minimization And Voltage Stability Have Been Done [1], While Transmission Loss Minimization And Power Installation Cost Using Evolutionary Computation For Improvement Of Voltage Stability Have Been Looked Into [2]. In Addition To Active Power Losses, Series Reactive Power Losses Of Transmission System Were Also Considered As One Of The Multiple Objectives. The Relationship Between Distance And Loadings On Power Losses Using The Existing 330KV Nigerian Transmission Network As A Case Study In His Empirical Modeling Of Power Losses As A Function Of Line Loadings And Lengths In The Nigeria 330KV Transmission Lines Has Been Considered [3]. A New Method For Calculating Transmission Power Losses Based On Exact Modeling Of Ohmic Loss Has Been Developed [4]. Mathematical Modeling To Study The Flow Of Power On Electric Power Transmission Lines Has Been Applied [7], Just To Mention A Few.

\section{Losses In Transmission Lines}

Losses Which Occur In Transmission Lines May Be Any Of These Three Types - Copper, Dielectric, And Radiation Or Induction Losses.

\section{1 copper Losses}

One Type Of Copper Loss Is I ${ }^{2} R$ Loss. In Rf Lines The Resistance Of The Conductors Is Never Equal To Zero. Whenever Current Flows Through One Of These Conductors, Some Energy Is Dissipated In Form Of 
Heat. This Heat Loss Is A Power Loss. With Copper Braid, Which Has A Resistance Higher Than Solid Tubing, This Power Loss Is Higher. Another Type Of Copper Loss Is Due To Skin Effect. When Dc Flows Through A Conductor, The Movement Of Electrons Through The Conductor's Cross Section Is Uniform. The Situation Is Somewhat Different When Ac Is Applied. The Expanding And Collapsing Fields About Each Electron Encircle Other Electrons. This Phenomenon, Called Self Induction, Retards The Movement Of The Encircled Electrons. The Flux Density At The Center Is So Great That Electron Movement At This Point Is Reduced. As Frequency Is Increased, The Opposition To The Flow Of Current In The Center Of The Wire Increases. Current In The Center Of The Wire Becomes Smaller And Most Of The Electron Flow Is On The Wire Surface. When The Frequency Applied Is 100 Megahertz Or Higher, The Electron Movement In The Center Is So Small That The Center Of The Wire Could Be Removed Without Any Noticeable Effect On Current. You Should Be Able To See That The Effective Cross-Sectional Area Decreases As The Frequency Increases. Since Resistance Is Inversely Proportional To The Cross-Sectional Area, The Resistance Will Increase As The Frequency Is Increased. Also, Since Power Loss Increases As Resistance Increases, Power Losses Increase With An Increase In Frequency Because Of Skin Effect. Copper Losses Can Be Minimized And Conductivity Increased In An Rf Line By Plating The Line With Silver. Since Silver Is A Better Conductor Than Copper, Most Of The Current Will Flow Through The Silver Layer. The Tubing Then Serves Primarily As A Mechanical Support.

\subsection{Dielectric Losses}

Dielectric Losses Result From The Heating Effect On The Dielectric Material Between The Conductors. Power From The Source Is Used In Heating The Dielectric. The Heat Produced Is Dissipated Into The Surrounding Medium. When There Is No Potential Difference Between Two Conductors, The Atoms In The Dielectric Material Between Them Are Normal And The Orbits Of The Electrons Are Circular. When There Is A Potential Difference Between Two Conductors, The Orbits Of The Electrons Change. The Excessive Negative Charge On One Conductor Repels Electrons On The Dielectric Toward The Positive Conductor And Thus Distorts The Orbits Of The Electrons. A Change In The Path Of Electrons Requires More Energy, Introducing A Power Loss. The Atomic Structure Of Rubber Is More Difficult To Distort Than The Structure Of Some Other Dielectric Materials. The Atoms Of Materials, Such As Polyethylene, Distort Easily. Therefore, Polyethylene Is Often Used As A Dielectric Because Less Power Is Consumed When Its Electron Orbits Are Distorted.

\subsection{Radiation And Induction Losses}

Radiation And Induction Losses Are Similar In That Both Are Caused By The Fields Surrounding The Conductors. Induction Losses Occur When The Electromagnetic Field About A Conductor Cuts Through Any Nearby Metallic Object And Current Is Induced In That Object. As A Result, Power Is Dissipated In The Object And Is Lost. Radiation Losses Occur Because Some Magnetic Lines Of Force About A Conductor Do Not Return To The Conductor When The Cycle Alternates. These Lines Of Force Are Projected Into Space As Radiation And This Result In Power Losses. That Implies Power Supplied From The Source Is Not Fully Getting To The Load.

\section{Method Used To Minimize Power Loss}

In This Research Work Capacitor Was Used To Generate Capacitive Power To Cancel The Inductive Reactance Of The System As Such Compensating For Power Losses Along A Medium Transmission Line Such That Power Generated From The Source Could Get To The End Users With Minimized Or No Loss.

\subsection{Series Capacitors}

Series Capacitors Are Generally Applied To Compensate The Excessive Inductance Of Long Transmission Lines, In Order To Reduce The Line Voltage Drop, Improve Its Voltage Regulation, Minimize Losses By Optimizing Load Distribution Between Parallel Transmission Lines, And To Increase The Power Transfer Capability. Similarly, Series Capacitors Are Also Installed In Electrical Power Systems To Improve Its Voltage Stability.

In Contrast To A Shunt Capacitor, Series Compensation Results In A Voltage Rise At The End Of The Feeder That Varies Directly With Load Current. In Other Words, Voltage Rise Is Zero At No Load And Maximum At Full Load. Consequently, Series Capacitors Do Not Need To Be Switched In Response To Changes In Power Demand. In Addition, A Series Capacitor Will Require Relatively Smaller KVAR And KV Ratings Than A Shunt Capacitor Providing An Equivalent Voltage Regulation. 


\subsection{Working Principle}

Series Capacitors Positively Affect The Voltage And Reactive Power Balance. When The Load Current Passes Through The Capacitors, The Voltage Drop Over The Capacitor Varies In Proportion To The Current. The Voltage Drop Is Capacitive, Such That It Offsets The Inductive Voltage Drop, Which Also Varies With The Load Current. The Result Is An Automatic Stabilizing Effect On The Voltage In The Network. At The Same Time, Series Capacitors Generate Reactive Capacitive Power, Which Improves The System Power Factor Resulting To Reduced Line Current And Line Losses And The Increase Of Load Capacity. The Generated Reactive Capacitive Power Varies Proportionally To The Square Of The Load Current. Thus, The Reactive Power Is Automatically Regulated.

\subsection{Applications}

Series Capacitors Can Correspondingly Find Purpose At All High-Impedance And High-Current Loads Such As, But Not Limited To The Following:

- Transmission Lines Of 400km And More For A Radial Line, And 800km And More For A Symmetrical Line.

- High Voltage Distribution Network That Has A High Series Inductive Reactance To Improve Its Receiving-End Voltage.

- Electric Arc And Resistance Welding Transformers In Cases Like Spot, Seam And Butt Welding.

- Induction Furnace, Where The Heating Is Due To Eddy Current Losses Induced By The Magnetic Field.

- Large Scale Electrolysis Of Aluminum, Copper Or Zinc.

- Electric Arc Furnace, Where Heating Is Caused By Arc Plasma Between The Two Electrodes. The Arcing Makes The Circuit Highly Inductive, Besides Generating Unbalanced Currents (Third Harmonics), Due To Different Touchdown Arc Distances In The Three Electrodes Which Make It A Non

Linear Impedance Load.

- Large Scale Electrolysis Of Aluminum, Copper Or Zinc.

- Mitigation Of Voltage Fluctuations (Flicker).

\subsection{Disadvantages And Cautions}

There Are Several Concerns That Must Be Evaluated In The Application Of Series Capacitors. The Following Disadvantages Limit The Application Of Series Capacitors On The Electric Power Systems:

- They Cannot Provide Reactive Compensation For Feeder Loads And Do Not Significantly Reduce System Losses.

- Series Capacitors Can Only Release Additional System Capacity If It Is Limited By Excessive Line Voltage Drop. On The Other Hand, Shunt Capacitors Are Effective When System Capacity Is Limited By High Line Current As Well.

- They Cannot Tolerate Fault Current. This Would Result To An Overvoltage And Should Be Prevented By Bypassing The Capacitor Through An Automatic Switch. In Addition, An Arrester Must Be

Connected Across The Capacitor To Divert Current Until The Switch Closes.

- Resonance And/Or Hunting With Synchronous And Induction Motors.

- Ferroresonance

\subsection{Capacitor Bank}

A Capacitor Bank Is Very Essential Equipment Of An Electrical Power System. The Power Required To Run All The Electrical Appliances And Load As Useful Power Is Active Power. The Active Power Is Expressed In KW Or MW. The Maximum Load Connected To The Electrical Power System Is Mainly Inductive In Nature Such As Electrical Transformers, Induction Motors, Synchronous Motor, Electric Furnaces, Fluorescent Lighting Are All Inductive In Nature. In Addition To These, Inductance Of Different Lines Also Contributes Inductance To The System. Because Of These, Inductance Of Different Lines Also Contributes Inductance To The System. Because Of Inductances, The System Current Lags Behind System Voltage. As The Lagging Angle Between Voltage And Current Increases, The Power Factor Of The System Draws More Current From The Source. More Electric Current Causes More Line Losses. Poor Electrical Power Factor Causes Poor Voltage Regulation. So To Avoid These Difficulties, The Electrical Power Factor Of The System Need To Be Improved. Capacitor Causes Current To Lead The Voltage, Capacitive Can Be Used To Cancel The Inductive Reactance Of The System. The Capacitor Reactance Is Generally Applied To The System By Using Static Capacitor In Shunt Or Series With The System. Instead Of Using A Single Unit Of Capacitor Per Phase Of The System, It Is Better To Use Bank Of Capacitor Units, In View Of Maintenance And Erection. This Group Or 
Bank Of Capacitor Is Known As CAPACITOR BANK. There Are Mainly Two Categories Of Capacitor Bank This Is Determined According To Their Connection Arrangements:

1) Shunt Capacitor.

2) Series Capacitor.

However, The Shunt Capacitor Is Very Commonly Used.

3.6 Calculation Of Ratings Of Capacitor Required For The Capacitor Bank.

The Size Of The Capacitor Used For The Capacitor Bank Is As Shown On The Excel Sheet On Page 6 Below With The Parameters Used Well Spelt Out.

\subsection{Location Of Capacitor Bank}

Theoretically It Is Always Desired To Commission Capacitor Bank Nearer To The Load. This Makes Transmission Of Reactive KVARS To Be Removed From A Greater Part Of The Network. Moreover If Capacitor And Load Are Connected Simultaneously, During Disconnection Of Load, Capacitor Is Also Disconnected From The Rest Of The Circuit. Hence, There Is No Question Of Over Compensation. But Connecting Capacitor With Each Individual Load Is Not Practical From The Economical Point Of View, As The Size Of Load Is Extremely Differ For Different Consumers. So Various Sizes Of Capacitors Are Not Always Readily Available. Hence, Proper Compensation Is Not Possible At Each Loading Point. Again Each Load Is Not Connected With System For 24 X 27 Hours. So The Capacitor Connected To The Load Can Not Be Fully Utilized. Therefore, Capacitor Is Not Installed For Small Load But For Medium And Large Load. Capacitor Bank Can Be Installed At Consumer Own Premises. Although The Inductive Loads Of Medium And Large Bulk Consumers Are Compensated, But Still There Would Be Considerable Amount Of VAR Demand Originated From Different Uncompensated Small Loads Connected To The System. In Addition To The Inductive Of Lines, Transformers Also Contribute VAR To The System. In View Of These Difficulties Instead Of Connecting Capacitor To Each Load, Large Capacitor Bank Is Installed At The Main Distribution Substation Or Secondary Grid Substation.

\subsection{Connection Of Shunt Capacitor Bank}

The Capacitor Bank Can Be Connected To The System Either In Delta Of In Star. In Star Connection, The Neutral Point May Be Grounded Or Not Depending Upon Protection Scheme For Capacitor Bank Adopted. In Some Cases The Capacitor Bank Is Formed By Double Star Formation. Generally, Large Capacitor Bank In Electrical Substation Is Connected In Star And They Are Quite Reliable.

\section{Conclusion}

Accurate Loss Minimization On High Voltage Transmission Line Is An Important Factor For The Efficiency Of Electric Power Network In Any Part Of The World, And Nigeria Can Not Be An Exception. In This Paper, The Use Of Capacitor Bank To Generate Capacitive Power Which In Turn Cancels The Inductive Reactance Of The System To Compensate For Line Losses Has Been Applied. This Is Mainly Used At Substations Along Transmission Lines For Effective Power Delivery.

\section{References}

[1]. Zakariya (2010): Comparison Between The Corona Power Loss Associated With HVDC Transmission Lines And The Ohmic Power Loss.

[2]. Abdullah Et Al (2010): Transmission Loss Minimization And Power Installation Cost Using Evolutionary Computation For Improvement Of Voltage Stability.

[3]. Onohaebi And Odiase (2010): The Relationship Between Distance And Loadings On Power Losses.

[4]. Moghadam And Berahmandpour (2010): Calculation Of Transmission Power Losses Base On Exact Modelling Of Ohmic Loss.

[5]. Thabendra Et Al (2009): Multi-Objective Optimization Methods For Power Loss Minimization And Voltage Stability.

[6]. Numphetch Et Al (2011): Loss Minimization Using Optimal Power Flow Based On Swarm Intelligences.

[7]. Oke, Michael Olufemi (2012): Minimization Of Power Losses Over Electric Power Transmission Lines.

[8]. Alexander, G.E. (N.D.). Series Compensated Line Protection - A Practical Evaluation Dugan, R., Mcgranaghan, M., Santoso, S., And Beaty, H.W. (2004). Electrical Power Systems Quality ( $2^{\text {nd }}$ Editon). New York: Mcgraw-Hill. 